\title{
SPIN PROPERTIES OF ELECTRONS IN A QUANTUM TUBE
}

\author{
A. Dargys \\ Semiconductor Physics Institute, A. Goštauto 11, LT-01108 Vilnius, Lithuania \\ E-mail: dargys@pfi.lt
}

Received 15 November 2007; revised 27 December 2007; accepted 22 February 2008

\begin{abstract}
Spin properties of electrons, the wave function of which is confined by cylindrical potential of a hollow semiconducting cylinder (quantum tube), and which simultaneously propagate along the cylinder axis are analysed. The spin-orbit interaction is included via Rashba and Dresselhaus Hamiltonians. The electron spin surface, which describes all possible spin eigenand superposition states, is shown to reduce to the Bloch sphere, independent of the electron energy and semiconductor band parameters. The electron dispersion can be tuned to a regime that is favourable for the operation of the spin-FET by trimming the diameter of the quantum tube.
\end{abstract}

Keywords: 85.75.-d, 71.70.Ej, 85.35.Be

PACS: spintronics, spin-orbit coupling, quantum well devices

\section{Introduction}

Nonplanar 2D electronic systems, where the electron moves on a curved surface, recently have attracted the attention of the experimentalists and theorists. A single-wall carbon nanotube probably is the best-known system of this kind (see, for example, books $[1,2]$ and review articles [3-5] on recent achievements in electrical, thermal, and spin properties of carbon nanotubes). The self-rolling of thin strained semiconductor bilayer systems, as proposed by Prinz et al. [6-8], is another example. The latter nanotubes support the electron motion on a curved surface in both elementary and compound semiconductors. A typical silicon-germanium nanotube may have a $230 \mathrm{~nm}$ diameter and be as long as $12 \mathrm{~mm}$. The nanotubes can be formed from thin solid films of almost any material deposited on a substrate, once one edge of the film is released from the substrate by a selective etching procedure. The geometric properties of such nanotubes are determined by mechanical strain in the layer and the etching time.

Of a general interest is how the global topology of a nanostructure affects the quantum-mechanical wave function, electron motion trajectories, and spin properties. The confinement topology of the quantum tube (QT) is different from that of a planar quantum well or quantum wire (rod). In the QT the electron is confined to tube wall and thus its ground state is characterized by zero or close to zero probability of being at the centre of the tube, Fig. 1. Here we shall be interested in the effect of spin-orbit (SO) interaction on electron spin properties in the semiconducting QTs. Ballistic transport and SO interaction of $2 \mathrm{D}$ electrons confined to cylindrical surface were considered theoretically in Refs. $[9,10]$, where it was shown that the transport in QTs, in contrast to planar 2D systems, depends on the sign of SO interaction constant. In [11] the $\mathbf{k} \cdot \mathbf{p}$ theory was developed for $2 \mathrm{D}$ holes in cylindrical quantum systems. In the present paper, general properties of electron spin in QT are analysed using a notion of the spin surface and a vectorial model for spin precession. In Sec. 2 the needed Hamiltonian is constructed and in Sec. 3 the electron spin properties are investigated. The properties of spin surfaces in planar quantum wells were discussed earlier in Refs. [12-15].

\section{The Hamiltonian}

Figure 1(a) shows the cross-section of the QT and its natural reference system, cylindrical coordinate system $(r, \varphi, z)$, where $r$ is the radius, $\varphi$ is the azimuthal angle, and the coordinate $z$ runs along the QT axis. We shall assume that the wall of the QT is thin enough and thus the QT can be characterized by an average radius $R$. 


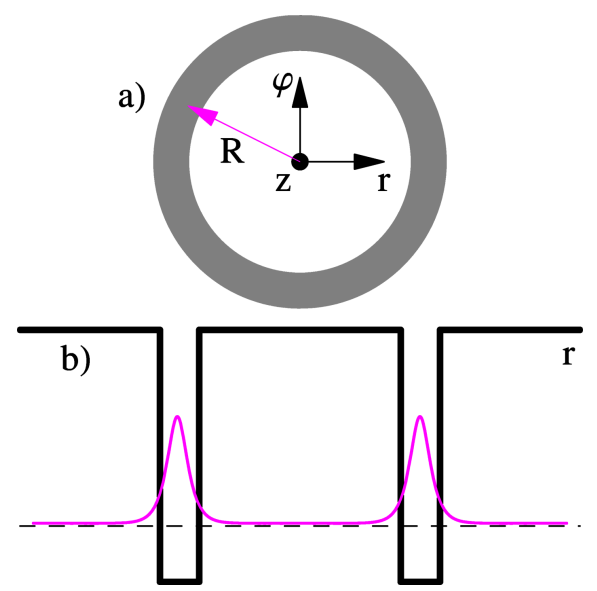

Fig. 1. (a) Cross-section of the cylindrical QT with the coordinate system used. (b) Profile of the potential energy in the direction perpendicular to QT axis and the ground state wave function.

The wave vector in the cylindrical coordinate system then reads

$$
\mathbf{k}=\left(k_{r}, k_{\varphi}, k_{z}\right)=\left(\left|\sqrt{k_{x}^{2}+k_{y}^{2}}\right|,-\frac{\mathrm{i}}{R} \frac{\partial}{\partial \varphi}, k_{z}\right),
$$

where $k_{x}, k_{y}, k_{z}$ are Cartesian wave vector components. In the following, the electron spin will be described by the vector of Pauli matrices $\sigma$, which in the cylindrical coordinate system has the following components:

$$
\begin{aligned}
\sigma_{r} & =\left(\begin{array}{cc}
0 & \mathrm{e}^{-\mathrm{i} \varphi} \\
\mathrm{e}^{\mathrm{i} \varphi} & 0
\end{array}\right), \\
\sigma_{\varphi} & =\left(\begin{array}{cc}
0 & -\mathrm{ie}^{-\mathrm{i} \varphi} \\
\mathrm{ie}^{\mathrm{i} \varphi} & 0
\end{array}\right), \\
\sigma_{z} & =\left(\begin{array}{cc}
1 & 0 \\
0 & -1
\end{array}\right) .
\end{aligned}
$$

These matrices satisfy the standard commutation relations.

In the stationary state the electron has the energy $E$ and propagates along the tube with the wave vector $k_{z}$. It can be described by a two-component spinor

$$
\boldsymbol{\Psi}=\boldsymbol{\psi}(r, \varphi) \mathrm{e}^{\mathrm{i} k_{z} z} \mathrm{e}^{-\mathrm{i} E t / \hbar},
$$

where $\psi(r, \varphi) \equiv \boldsymbol{\psi}$ takes into account the electron spin. In the following the coordinate $r$ will be suppressed, keeping in mind that only the fundamental radial mode has been selected. The other notations are standard. The confining potential $V(r)$ is assumed to have a cylindrical symmetry with potential minimum at $r \approx R>0$. Figure 1(b) shows a possible potential profile which is equal zero everywhere, $V(r)=0$, except in a cylindrical shell, where it is constant: $V(r)=$ const $<0$. In the limiting case of an infinite confinement the electron wave functions will be bound to the cylindrical shell. It can be shown that in the latter case all radial modes will propagate with the same velocity proportional to $k_{z}$. For an arbitrary potential distribution there may be a dispersion between the different radial modes. In the following we shall assume that the confinement is strong enough and, therefore, the energy of the fundamental mode is well separated from the higher order modes. A typical probability distribution of the fundamental mode is shown in Fig. 1(b).

Under the above assumptions and with the SO interaction included, the eigenvalue equation for the twocomponent spinor $\psi$ assumes the form

$$
-\frac{\partial^{2} \boldsymbol{\psi}}{\partial \varphi^{2}}+k^{2} \boldsymbol{\psi}+H_{\mathrm{SO}} \boldsymbol{\psi}=\varepsilon \boldsymbol{\psi},
$$

where the Hamiltonian $H_{\text {SO }}$ takes into account the SO interaction. It contains nondiagonal matrix elements which mix up the spin-up and spin-down states of $\boldsymbol{\psi}$. Two kinds of SO interaction [16] that are characteristic of semiconducting nanostructures will be included. The Rashba interaction, which is proportional to the constant $\alpha$, comes from the structural asymmetry. In the case of QT, the latter comes from the fact that the inner and outer surfaces of the confining shell are not equivalent, for example, their curvature is different, or the lattice strains in the inner and outer surfaces of the wall are not equal, etc. The Dresselhaus SO interaction, which is proportional to the constant $\beta$, comes from the absence of the inversion symmetry in the lattice of the constituent material. Due to large strains in nanotubes, the inversion asymmetry may be induced in otherwise centrosymmetric materials such as silicon. In the following the dimensionless energy, wave vector, Rashba and Dresselhaus constants will be used:

$$
\begin{gathered}
\varepsilon=\frac{2 m^{*} R^{2} E}{\hbar^{2}}, \quad k=R k_{z}, \\
\tilde{\alpha}=\frac{2 \alpha m^{*} R}{\hbar^{2}}, \quad \tilde{\beta}=\frac{2 \beta m^{*} R}{\hbar^{2}},
\end{gathered}
$$

where $m^{*}$ is the effective mass.

The spinor should satisfy the periodic boundary condition on a circumference of the QT, $\psi(\varphi)=\psi(\varphi+$ $2 \pi)$. Since the projection of the total angular momentum is equal to sum of orbital and spin components, $L_{z}+s_{z}=L_{z} \pm \frac{1}{2} \equiv j$, the eigenspinors should satisfy the following periodic boundary conditions:

$$
\boldsymbol{\psi}(\varphi)=\left(\begin{array}{c}
\mathrm{e}^{\mathrm{i}\left(j-\frac{1}{2}\right) \varphi} \psi_{f} \\
0
\end{array}\right) \text { or }\left(\begin{array}{c}
0 \\
\mathrm{e}^{\mathrm{i}\left(j+\frac{1}{2}\right) \varphi} \psi_{f}
\end{array}\right)
$$


where the projection $j$ assumes the values $j= \pm \frac{1}{2}, \pm \frac{3}{2}$, $\pm \frac{5}{2}, \ldots$ and $\psi_{f}$ is independent of the azimuthal angle $\varphi$.

The total Hamiltonian of the Eq. (6) consists of kinetic, Rashba, and Dresselhaus contributions,

$$
H=H_{\mathrm{k}}+H_{\mathrm{R}}+H_{\mathrm{D}} .
$$

The kinetic Hamiltonian in the basis (9) is diagonal. In the normalized form it assumes the form

$$
H_{\mathrm{k}}=\left(\begin{array}{cc}
k^{2}+\left(j-\frac{1}{2}\right)^{2} & 0 \\
0 & k^{2}+\left(j+\frac{1}{2}\right)^{2}
\end{array}\right) .
$$

It includes the electron motion along and around the cylinder of radius $R$.

In coordinate independent notation the Rashba Hamiltonian is given by [16]

$$
H_{\mathrm{R}}=\alpha(\boldsymbol{\sigma} \times \mathbf{k}) \cdot \mathbf{n},
$$

where $\mathbf{n}$ is the normal to the interface, in our case to the QT surface. In the cylindrical coordinates, using the matrices (2)-(4) one finds

$$
H_{\mathrm{R}}=\alpha\left(\sigma_{\varphi} k_{z}-\sigma_{z} k_{\varphi}\right)=\alpha\left(\begin{array}{cc}
-k_{\varphi} & -\mathrm{ie}^{-\mathrm{i} \varphi} k_{z} \\
\mathrm{ie}^{\mathrm{i} \varphi} k_{z} & k_{\varphi}
\end{array}\right) .
$$

The angular wave vector $k_{\varphi}$ is given by Eq. (1). In $H_{\mathrm{R}}$, in addition, we have taken into account a possible rotation of the coordinate system around the $z$ axis by including the exponent $\mathrm{e}^{\mathrm{i} \varphi}$. As we shall see, the eigenenergies are invariant to such rotation. With the exponents included, some of the intermediate calculations appear to be simpler.

In coordinate independent representation the Dresselhaus Hamiltonian

$$
H_{\mathrm{D}}=\gamma \boldsymbol{\sigma} \cdot \boldsymbol{\kappa}
$$

is proportional to the third power of the wave vector components. In the Cartesian coordinate system the components of the vector $\boldsymbol{\kappa}$ are $\kappa_{x}=k_{x}\left(k_{y}^{2}-k_{z}^{2}\right)$, $\kappa_{y}=k_{y}\left(k_{z}^{2}-k_{x}^{2}\right), \kappa_{z}=k_{z}\left(k_{x}^{2}-k_{y}^{2}\right)$. In the case of $2 \mathrm{D}$ planar quantum well the Hamiltonian $H_{\mathrm{D}}$ can be simplified if one neglects the anisotropy in SO splitting [17]. The simplified Hamiltonian, which is frequently used in the analysis, becomes proportional to the first rather than third power of wave vector components. We shall use the simplified form of the Hamiltonian. When the heterostructure interface is perpendicular to the $z$ axis the Hamiltonian (14) simplifies to

$$
H_{\mathrm{D}} \approx \beta\left(\sigma_{x} k_{x}-\sigma_{y} k_{y}\right) .
$$

Note that in the 2D case a different SO interaction constant has appeared. When the $z$ axis coincides with the

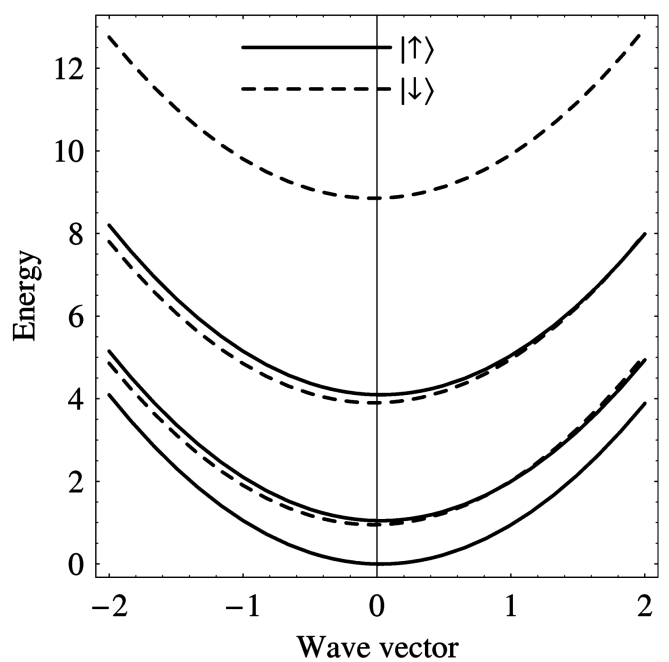

Fig. 2. Dispersion (normalized energy versus normalized wave vector) calculated with formula (18) at SO parameter values $\tilde{\alpha}=\tilde{\beta}=$ 0.05 and $j=\frac{1}{2}, \frac{3}{2}$, and $\frac{5}{2}$. The solid and dashed lines correspond to up and down eigenstates. The larger the angular momentum $j$ is, the higher is the energy of the corresponding curve.

QT symmetry axis, the simplified Dresselhaus Hamiltonian (15) in the cylindrical coordinate system reads

$$
H_{\mathrm{D}}=\beta\left(\left\{\sigma_{\varphi}, k_{\varphi}\right\}-\sigma_{z} k_{z}\right),
$$

where the curly brackets indicate the symmetrized product of the operators, $\left\{\sigma_{\varphi}, k_{\varphi}\right\}=\left(\sigma_{\varphi} k_{\varphi}+k_{\varphi} \sigma_{\varphi}\right) /$ 2. Then, in the basis (9) the normalized Dresselhaus Hamiltonian assumes the following form:

$$
H_{\mathrm{D}}=\tilde{\beta}\left(\begin{array}{cc}
-k & -\mathrm{i} j \mathrm{e}^{-\mathrm{i} \varphi} \\
\mathrm{i} j \mathrm{e}^{\mathrm{i} \varphi} & k
\end{array}\right) .
$$

Similarly as in the Rashba Hamiltonian (13), here we have included the exponents in the off-diagonal terms.

The spectrum of the total Hamiltonian $H=H_{\mathrm{k}}+$ $H_{\mathrm{R}}+H_{\mathrm{D}}$ is

$$
\begin{aligned}
\varepsilon= & j^{2}+k^{2}+\frac{1-2 \tilde{\alpha}}{4} \\
& \pm \sqrt{j(j-2 j \tilde{\alpha}+2 k \tilde{\beta})+\left(j^{2}+k^{2}\right)\left(\tilde{\alpha}^{2}+\tilde{\beta}^{2}\right)},
\end{aligned}
$$

where the plus and minus signs before the square root, as we shall see, correspond to two spin eigenstates ${ }^{1}$. Figure 2 shows the character of the spectrum for three subbands that correspond to the lowest values of the total angular momentum: $j=\frac{1}{2}, \frac{3}{2}$, and $\frac{5}{2}$. For negative

${ }^{1}$ In the Ref. [10] an additional cross term $-4 j k \tilde{\alpha} \tilde{\beta}$ under the root of the spectrum is present. This term reappears if the Dresselhaus Hamiltonian $H_{\mathrm{D}}$ is replaced by its complex conjugate. However in this case the Rashba (13) and Dresselhaus (17) Hamiltonians are in different coordinate systems, mutually rotated around the $z$ axis by the angle $\pi$. 
$j$ 's (rotation in the opposite direction around the circumference of the QT) the dispersions are mirror images with respect to the vertical axis. The parameters used in the calculation correspond to realistic values: $\alpha=\beta=2.3 \cdot 10^{-10} \mathrm{eV} \mathrm{cm}, m^{*} / m_{0}=0.066$, and $R=$ $50 \mathrm{~nm}$. For these parameter values the energy normalization unit is $9.1 \mathrm{meV}$.

\section{Spin surfaces and spin trajectories}

In the formulation of the problem it has been tacitly assumed that the quantization axis is parallel to QT axis, i. e., of the three Pauli matrices only $\sigma_{z}$ is diagonal. However, as we shall see, in the presence of SO interaction the direction of the natural quantization axis is different. The spin surface represents all possible directions and magnitudes of the average spin in the spin space $[12,15,18,19]$. In the simplest case when the $\mathrm{SO}$ interaction is unimportant it reduces to the Bloch sphere [18]. There is one-to-one correspondence between the points on the spin surface and all possible superpositions in the Hilbert space. The straight line that connects "north" and "south" poles (see Fig. 3) on the spin surface corresponds to the natural quantization axis. By this reason the evolution of a spin state in the Hilbert space under the control field is fully reflected on the spin surface in a three-dimensional spin space. The latter is more transparent and intuitively more acceptable [15]. Furthermore, the points on the spin surface are directly related to the magnetization of the spin system.

To obtain the spin surface the two-dimensional spinor was parametrized in the energy representation,

$$
\boldsymbol{\psi}_{\varepsilon}=\left(\mathrm{e}^{\mathrm{i} \phi / 2} \cos \vartheta, \mathrm{e}^{-\mathrm{i} \phi / 2} \sin \vartheta\right),
$$

which then was transformed to the initial representation, where the quantization axis is parallel to $z$ axis, by an appropriate unitary matrix. The latter was constructed from the eigenvectors of the total Hamiltonian (10). The spin surface can be drawn in the spin space by visualizing the average spin

$$
\langle\boldsymbol{\sigma}(\vartheta, \phi)\rangle=\langle\boldsymbol{\psi}(\vartheta, \phi)|\boldsymbol{\sigma}| \boldsymbol{\psi}(\vartheta, \phi)\rangle,
$$

where the free parameters $\vartheta$ and $\phi$ are varied in an appropriate range. When both the Rashba and Dresselhaus Hamiltonians are included, the spin surface after somewhat lengthy calculations can be expressed in the cylindrical coordinate system by the components

$$
\left\langle\sigma_{r}\right\rangle=-\sin \phi \sin 2 \vartheta,
$$

$$
\begin{gathered}
\left\langle\sigma_{\varphi}\right\rangle=-d^{-1}[(j-j \tilde{\alpha}+k \tilde{\beta}) \cos \phi \sin 2 \vartheta \\
+(k \tilde{\alpha}+j \tilde{\beta}) \cos 2 \vartheta] \\
\left\langle\sigma_{z}\right\rangle=d^{-1}[(j-j \tilde{\alpha}+k \tilde{\beta}) \cos 2 \vartheta \\
-(k \tilde{\alpha}+j \tilde{\beta}) \cos \phi \sin 2 \vartheta)]
\end{gathered}
$$

where $d=\sqrt{j(j-2 j \tilde{\alpha}+2 k \tilde{\beta})+\left(j^{2}+k^{2}\right)\left(\tilde{\alpha}^{2}+\tilde{\beta}^{2}\right)}$. The spin surface depends on the wave vector magnitude, Rashba and Dresselhaus constants. However, the important property is that the square of the average spin vector, or the radius of the spin surface, appears to be independent of all parameters and exactly equals to unity:

$$
\langle\boldsymbol{\sigma}\rangle^{2}=1 .
$$

From this result a very important conclusion follows: after the Rashba and Dresselhaus SO interactions have been included, the spin surface remains spherical and thus in all cases the electron spin state can be represented on the Bloch sphere. It should be remembered that in the presence of SO interaction the spin is not a good quantum number and therefore, in general, the spin surface should not necessarily reduce to spherical. Examples of nonspherical spin surfaces in the cases of planar quantum wells can be found in Refs. [13-15]. Figure 3 shows the spin surface, where the parallels correspond to $\phi=$ const and meridians to $\vartheta=$ const. The straight line that connects the poles represents the natural or true quantization axis of the problem. The precession of the average spin, or macroscopic magnetization vector, takes place around this axis. Although the spin surface appears to be spherical, a concrete direction of the quantization axis depends on band parameters, electron wave vector, and QT diameter. Below, the required dependence will be found.

The equation of motion for the spin operator $\sigma$ can be expressed through the commutator

$$
\frac{\mathrm{d} \boldsymbol{\sigma}}{\mathrm{d} t}=\frac{\mathrm{i}}{\hbar}[\boldsymbol{\sigma}, H],
$$

where $H$ is the Hamiltonian. The measured spin $\langle\mathbf{S}\rangle$ is related to the averaged Pauli matrices via the relation $\langle\mathbf{S}\rangle=\hbar\langle\boldsymbol{\sigma}\rangle / 2$. If the Hamiltonian (10) and the parametrized wave function (19) transformed to the representation $\sigma_{z}$ are used, then it appears that the equation of motion depends only on the products of the following trigonometric functions entering the Eqs. (21)-(23):

$$
\sin \phi \sin 2 \vartheta=-\left\langle\sigma_{r}\right\rangle,
$$




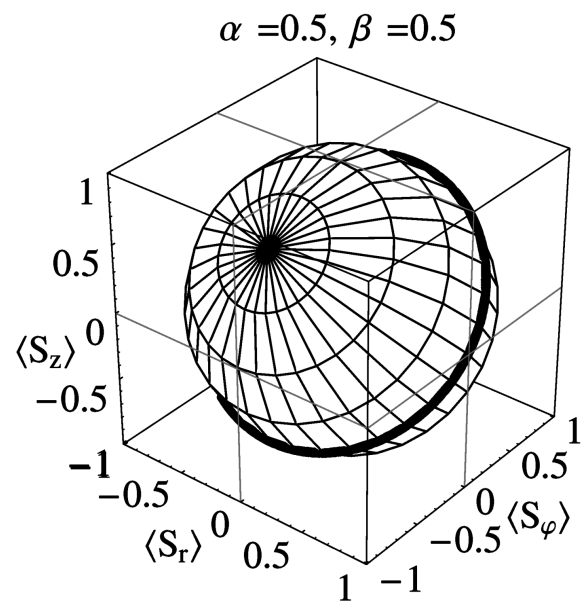

Fig. 3. Spin surface represented by parallels and meridians, and precession trajectories (thick line) that run near the equator for the Hamiltonian with Rashba and Dresselhaus contributions included: $\tilde{\alpha}=\tilde{\beta}=0.5$. The wave vector for various trajectories is in the range $k=0.7-1.5$ and $j=1 / 2$. The spin surface was drawn for $k=1$.

$$
\begin{array}{r}
\cos \phi \sin 2 \vartheta=-\frac{2}{\Omega}\left[(j-j \tilde{\alpha}+k \tilde{\beta})\left\langle\sigma_{\varphi}\right\rangle\right. \\
\left.+(k \tilde{\alpha}+j \tilde{\beta})\left\langle\sigma_{z}\right\rangle\right], \\
\cos 2 \vartheta=\frac{2}{\Omega}\left[(j-j \tilde{\alpha}+k \tilde{\beta})\left\langle\sigma_{z}\right\rangle\right. \\
\left.-(k \tilde{\alpha}+j \tilde{\beta})\left\langle\sigma_{\varphi}\right\rangle\right],
\end{array}
$$

where $\Omega$, as we shall see, can be identified with the precession frequency

$$
\begin{aligned}
\Omega= & |\boldsymbol{\Omega}|= \\
& 2 \sqrt{j^{2}(1-2 \tilde{\alpha})+\left(j^{2}+k^{2}\right)\left(\tilde{\alpha}^{2}+\tilde{\beta}^{2}\right)+2 j k \tilde{\beta}} .
\end{aligned}
$$

One can construct the system of differential equations that does not contain the parameters $\vartheta$ and $\phi$ by, at first, calculating the quantum mechanical average of the equation of motion (25) and then replacing the products of trigonometric functions by right-hand sides of (26)(28). Since this system of equations describes the precession of the tip of spin or magnetization vector on the Bloch sphere, it can be cast into a form of a vectorial differential equation, similar to the spinning top equation,

$$
\frac{\mathrm{d}\langle\mathbf{S}\rangle}{\mathrm{d} t}=-\boldsymbol{\Omega} \times\langle\mathbf{S}\rangle,
$$

where $\langle\mathbf{S}\rangle=\hbar\langle\boldsymbol{\sigma}\rangle / 2=\hbar\left(\left\langle\sigma_{r}\right\rangle,\left\langle\sigma_{\varphi}\right\rangle,\left\langle\sigma_{z}\right\rangle\right) / 2$ and where the averages are given by Eqs. (21)-(23), and the precession vector $\Omega=\left(\Omega_{r}, \Omega_{\phi}, \Omega_{z}\right)$ in the cylindrical coordinate system is given by

$$
\boldsymbol{\Omega}=2\left(\begin{array}{c}
(k \tilde{\alpha}+j \tilde{\beta}) \sin \varphi \\
(k \tilde{\alpha}+j \tilde{\beta}) \cos \varphi \\
j(1-\tilde{\alpha})+k \tilde{\beta}
\end{array}\right) .
$$

The module of the vector $\Omega$ gives the spin splitting energy (29), which coincides with the square root in the dispersion relation (18). It is seen that the variation of the polar angle $\varphi$ rotates the natural quantization axis around the QT axis. Since the spin splitting energy does not depend on $\varphi$, the appearance of $\varphi$ in (31) is a reflection of the cylindrical symmetry of the problem. Thus, a concrete direction of the quantization axis on the cone still remains undefined. Without losing generality it can be assumed that $\varphi=0$. In the latter case the quantization axis, as follows from $(31)$, lies in $(\varphi, z)$ plane. Thus, using the precession vector (31) the system of differential equations can be rewritten in a form of the vectorial equation for a spinning top. If needed the obtained spin precession equation (30) can be supplemented by a driving torque, or spin relaxation terms. The spherical symmetry of the spin surface assures that all imaginable trajectories of the magnetization vector end will lie on the Bloch sphere. The solid line on the equator in Fig. 3 shows the spin trajectories calculated with Eqs. (30) and (31) at wave vector values in the range $k=0.7-1.5$, and $j=\frac{1}{2}, \tilde{\alpha}=\tilde{\beta}=0.5$. The integration of the precession equation was performed with the initial condition $\Omega_{0}=(0,1,1) / \sqrt{2}$, which corresponds to $\vartheta=\pi / 4$ and $\phi=0$ in the parameter space. At employed parameter values, it is seen that the scatter of the trajectories is rather small.

Some of spin properties of the QT immediately follow from the precession equations (30), (31). Firstly, if $k \approx 0$, the precession frequency $\Omega$ is not symmetric with respect to sign of the Rashba constant. We shall remind that in a case of the planar quantum well only the absolute value of the Rashba coefficient appears. Thus, in the QT the sign of the SO interaction can be determined experimentally. Secondly, when the Rashba interaction dominates $(\tilde{\alpha} \gg \tilde{\beta})$, the expression (31) for $\boldsymbol{\Omega}$ shows that the natural quantization axis in this case does not depend on material parameters if, in addition, $\tilde{\alpha}=1$. The quantization axis in this case is perpendicular to QT axis. Since $\tilde{\alpha}=2 \alpha R / \hbar^{2}$, this regime can be realized by trimming the radius of the QT. When $\tilde{\alpha}=1$ and $\tilde{\beta}=0$, the precession frequency is proportional to $k$. This property is important for a proper operation of the spin-FET $[14,20]$, since in this case the trajectories 
of all electrons, independent of their energy, lie on the same spatial helix, in other words the precession length does not dependent on the energy of the injected electron. However, at present it appears difficult to satisfy the condition $\tilde{\alpha}=1$ at experimentally available values of the Rashba constant [21].

In this paper we have considered only single electron excitations. In one spatial dimension at high particle densities the Luttinger liquid is formed which has altogether different properties from three-dimensional Fermi liquid. The latter is stable with respect to electron-hole excitations above and below the Fermi sea, whereas the Luttinger liquid is unstable in this respect and can support only collective excitations. The remarkable feature of the Luttinger liquid is the separation between collective spin and charge oscillations at finite temperatures, what is altogether different from the single electron or hole excitations where the spin and charge are tied together. Since the spin-collective excitations have lower excitation energy, the so-called spinincoherent Luttinger liquid may be formed, where the collective charge excitations are near the ground state while spin excitations are highly excited, and thus are incoherent. This subject was recently discussed in the review article [22].

\section{Conclusion}

The main conclusion that follows from this study is that the spin surface is spherical, independent of SO interaction type, strength, and magnitude of the electron velocity along the QT. This indicates that, in principle, it is possible to inject the spin into the QT with an arbitrary direction of the spin. Of course, only those directions which coincide with the natural quantization or precession axis will be stationary. However, for spintronics devices the most interesting are the superpositions states. The deduced precession equations (30)-(31) allow one to model the evolution of such spin states. If the Rashba interaction predominates in the QT then by tuning the QT diameter it is possible to reach the regime favourable for spin-FET operation.

\section{Acknowledgement}

The work was partly supported by the Lithuanian State Science and Studies Foundation under contract C-07004.

\section{References}

[1] R. Saito, G. Dresselhaus, and M.S. Dresselhaus, Physical Properties of Carbon Nanotubes (Imperial College Press, London, 1999).

[2] P.N. D'yachkov, Carbon Nanotubes (Binom, Moscow, 2006) [in Russian].

[3] S. Ciraci, A. Boldum, and I.P. Batra, Quantum effects in electrical and thermal transport through nanowires, J. Phys. Cond. Matter 13(29), R537-R568 (2001).

[4] C. Schönenberger, Charge and spin transport in carbon nanotubes, Semicond. Sci. Technol. 21(11), S1-S9 (2006).

[5] A. Cottet, T. Kontos, S. Sahoo, H.T. Mann, M.-S. Choi, W. Belzig, C. Bruder, A.F. Morpurgo, and C. Schönenberger, Nanospintronics with carbon nanotubes, Semicond. Sci. Technol. 21(11), S78-S95 (2006).

[6] V.Ya. Prinz, V.A. Seleznev, A.K. Gutakovsky, A.V. Chehovskiy, V.V. Preobrazhenskii, M.A. Putyato, and T.A. Gavrilova, Free-standing and overgrown InGaAs/GaAs nanotubes, nanohelices and their arrays, Physica E 6(1), 828-831 (1984).

[7] O.G. Schmidt and K. Eberl, Thin solid films roll up into nanotubes, Nature 410(6825), 168 (2001).

[8] K.J. Friedland, R. Hey, H. Kostial, A. Riedel, and K.H. Ploog, Measurements of ballistic transport at nonuniform magnetic fields in cross junctions of a curved two-dimensional electron gas, Phys. Rev. B 75(4), 045347-1-4 (2007).

[9] L.I. Magarill, D.A. Romanov, and A.V. Chaplik, Twodimensional electron kinetics on a curved surface, JETP Lett. 64, 460 (1996) [Pis'ma Zh. Eksp. Teor. Fiz. 64(6), 421-426 (1996)].

[10] L.I. Magarill, D.A. Romanov, and A.V. Chaplik, Ballistic transport and spin-orbit interaction of twodimensional electrons on a cylindrical surface, JETP 86(4), 771 (1998) [Zh. Eksp. Teor. Fiz. 113(4), 1411$1428(1998)]$.

[11] P.C. Sercel and K.J. Vahala, Analytical formalism for determining quantum-wire and quantum-dot band structure in the multiband envelope-function approximation, Phys. Rev. B 42(6), 3690-3710 (1990).

[12] A. Dargys, Spin surfaces and trajectories in valence bands of tetrahedral semiconductors, Phys. Status Solidi B 241(1), 145-154 (2004).

[13] A. Dargys, Free-electron spin surfaces in 3D and 2D zinc-blende semiconductors, Phys. Status Solidi B 243(8), R54-R56 (2006).

[14] A. Dargys, Why the spin-FET does not work?, Lithuanian J. Phys. 47(2), 185-194 (2007).

[15] A. Dargys, Precession trajectories of the hole spin in zinc-blende semiconductors, Solid-State Electron. 51(1), 93-100 (2007).

[16] R. Winkler, Spin-Orbit Coupling Effects in TwoDimensional Electron and Hole Systems (SpringerVerlag, Berlin-Heidelberg, 2003). 
[17] R. Eppenga and M.F.H. Schuurmans, Effect of bulk inversion asymmetry on [001], [110], and [111] GaAs / AlAs quantum wells, Phys. Rev. B 37(18), 10923-10926 (1988).

[18] L. Allen and J.H. Eberly, Optical Resonance and TwoLevel Atoms (John Wiley and Sons, New York, 1975).

[19] A. Dargys, Coherent properties of hole spin, Lithuanian J. Phys. 43(2), 123-128 (2003).
[20] S. Datta and B. Das, Electronic analog of the electrooptic modulator, Appl. Phys. Lett. 56(2), 665-667 (1990).

[21] R. H. Silsbee, Spin-orbit induced coupling of charge current and spin polarization, J. Phys. Cond. Matter 16(7), R179-R207 (2004).

[22] G.A. Fiete, Colloquium: The spin-incoherent Luttinger liquid, Rev. Mod. Phys. 79(3), 801-820 (2007).

\title{
ELEKTRONŲ SUKINIO SAVYBĖS KVANTINIAME VAMZDELYJE
}

\author{
A. Dargys \\ Puslaidininkiu fizikos institutas, Vilnius, Lietuva
}

\begin{abstract}
Santrauka
Išnagrinètos elektrono sukinio savybės, kai elektrono banginė funkcija lokalizuota puslaidininkio cilindriniame potenciale (kvantinio vamzdelio sienelèje) ir kai elektronas gali judèti išilgai vamzdelio. Sąveika tarp elektrono sukinio ir jo orbitinio judejjimo įskaityta per Rašbos ir Dreselhauso hamiltonianus. Gauta, kad elektrono
\end{abstract}

sukinio paviršius - pastarasis nusako visas galimas savąsias ir superpozicines elektrono sukinio būsenas - transformuojasi į Blocho sferą, nepriklausomai nuo puslaidininkị nusakančiu parametrų bei elektrono energijos. Parodyta, kad, parinkus kvantinio vamzdelio diametrą, galima pasiekti režimą, kuris yra palankus sukinio tranzistoriaus darbui. 